[30] A. Bayliss and E. Turkel, "Radiation boundary conditions for wavelike equations," Comm. Pure Appl. Math., vol. 33, pp. 707-725, 1980.

[31] B. Engquist and A. Majda, "Absorbing boundary conditions for the numerical simulation of waves," Math. Comp., vol. 31, pp. 629-651, 1977.

[32] A. F. Peterson, "Absorbing boundary conditions for the vector wave equation," Microwave and Opt. Techn. Lett., vol. 1, pp. 62-64, Apr. 1988.

[33] W. C. Chew, Waves and Fields in Inhomogeneous Media. New York: Van Nostrand Reinhold, 1990, pp. 246-256.

[34] A. F. Peterson and S. P. Castillo, "A frequency-domain differential equation formulation for electromagnetic scattering from inhomogeneous cylinders," IEEE Trans. Antennas Propagat., vol. 37, pp. 601-607, May 1989.

[35] B. J. McCartin, L. J. Bahrmasel, and G. Meltz, "Application of the control region approximationto two-dimensional electromagneticscattering," in Finite Element and Finite Difference Methods in Electromagnetic Scattering, M. A. Morgan, Ed. New York: Elsevier, 1990 .

[36] J. D'Angelo and I. D. Mayergoyz, "Finite element methods for the solution of RF radiation and scattering problems," Electromagn., vol. 10, pp, 177-199, 1990 .

[37] J. Dongarra, J. R. Bunch, C. B. Moler, and G. W. Stewart, LINPACK User's Guide. Philadelphia, PA: SIAM, 1979.

[38] Z. Gong and A. W. Glisson, "A hybrid equation approach for the solution of electromagnetic scattering problems involving two-dimensional inhomogeneous dielectric cylinders," ZEEE Trans. Antennas Propagat., vol. 38, pp. 60-68, Jan. 1990.

[39] K. L. Wu, G. Y. Delisle, D. G. Fang, and M. Lecours, "Coupled finite element and boundary element methods in electromagnetic scattering," in Finite Element and Finite Difference Methods in Electromagnetic Scattering, M. A. Morgan, Ed. New York: Elsevier, 1990 .

[40] J. F. Lee, "New methods for predicting bistatic electromagnetic scattering from complicated and inhomogeneous shapes," M.S.dissertation, Camegie-Mellon University, Pittsburgh, PA, 1986.

[41] J. F. Lee and Z. Cendes, "Transfinite elements: a highly efficien procedure for modeling open-field problems," J. Appl. Phys., vol. 61, pp. 3913-3915, Apr. 1987.

\section{A Note on Spatially Weighted Subarray Covariance Averaging Schemes}

\author{
K. J. Raghunath and V. U. Reddy
}

Abstract-In spatial smoothing, the effective correlation among the impinging sources goes to zero only asymptotically with the number of subarrays. Recently, Takao et al. and Paulraj et al. proposed Toeplitzization and adaptively weighted subarray covariance averaging methods, respectively, wherein the impinging sources are perfectly decorrelated whenever the number of subarrays exceeds a minimum value. While both methods achieve the same result, the formulations of the two methods are different. In this communication, we first suggest an alternate formulation of the same problem and then compare the three methods, and show that the solutions obtained by them are equivalent.

\section{INTRODUCTION}

Subspace based algorithms such as MUSIC [3] and minimum variance beamformers such as Capon's beamformer [4] break down when the impinging sources are coherent. For the case of a uniform linear array, spatial smoothing [5], [6] has been proposed to decor.

Manuscript received July 25, 1990; revised February 18, 1992 The authors are with the Department of Electrical Communication Engineering, Indian Institute of Science, Bangalore 560012, India.

IEEE Log Number 9200423 relate the impinging sources thereby removing the effects due to correlation. In beamforming, however, any residual correlation between the sources will affect the performance. It is shown in [7] that this correlation decreases to zero only asymptotically with the number of subarrays.

Weighted spatial smoothing schemes have been recently suggested, wherein the smoothed matrix is obtained by taking a weighted average of the subarray covariances. The weights are chosen such that the sources are perfectly decorrelated. Two such methods are the Toeplitzization method of Takao et al. [1] and the adaptively weighted subarray covariance averaging scheme of Paulraj et al. [2]. Both of these methods force the smoothed covariance matrix to Toeplitz form, since for a uniform linear array, the array covariance matrix is in Toeplitz form when the sources are uncorrelated.

While both methods achieve the same result, the formulation of the schemes is different. In the method of Paulraj et $a l$. [2] it is assumed that the directions of arrival (DOA's) of all the correlated sources are known a priori. Their simulation results, however, show that the approximate knowledge of the DOA's is good enough to give sufficiently accurate solutions. Further, in this method the excess degrees of freedom are utilized to minimize the norm of the weighting coefficients. The method of Takao et al. [1], on the other hand, works only with the subarray covariance matrices and requires no knowledge of the DOA's. In their method too, as we show later in Section IV, any available excess degrees of freedom are utilized to control the norm of the weight vector, though this fact was not brought out explicitly in [1]. Though the value of the norm has no bearing in the asymptotic case (i.e., the case with infinite data or true covariance), it influences the performance of both MUSIC and the minimum variance beamformer when only a finite number of snapshots are available for estimating the array covariance matrix.

In this communication, we first suggest an alternative formulation of the same problem and then show that the solutions obtained by all three methods, i.e., methods of [1], [2] and the alternative method, are equivalent. We may point out here that our results are given for the asymptotic case.

\section{BACKGROUND}

Consider a uniform linear array of $\boldsymbol{m}$ sensors. The signals received from the array, stacked in a vector form, can be written as

$$
\mathbf{r}(t)=A \mathbf{s}(t)+\mathbf{n}(t)
$$

with

$$
A=\left[\mathrm{a}\left(\theta_{1}\right), \cdots, \mathfrak{a}\left(\theta_{d}\right)\right]
$$

where $\mathbf{s}(\cdot)$ is the source signal vector, $\mathbf{n}(\cdot)$ is the additive white noise, $\mathbf{a}\left(\theta_{k}\right)$ is the steering or direction vector corresponding to the direction $\theta_{k}$, and $d$ is the number of sources. For the uniform linear array, with isotropic sensors and interelement spacing $\mathbf{A}$, and assuming the first sensor to be the reference sensor, the steering vector can be written as

$$
\mathbf{a}\left(\theta_{k}\right)=\left[1, e^{-j w_{0} \tau_{k}}, \cdots, e^{-j w_{0}(m-1) \tau_{k}}\right]^{T}
$$

where

$$
\tau_{k}=\mathbf{A} \sin \theta_{k} / \mathcal{C}
$$

The array covariance matrix $\mathrm{R}$ is then given by

$$
\boldsymbol{R}=E\left[\mathbf{r}(t) \mathbf{r}^{+}(t)\right]=A S A^{+}+\sigma_{n}^{2} I
$$

where $\mathbf{S}$ is the source covariance matrix and $\sigma_{n}^{2}$ is the noise variance, and the superscript plus sign denotes the complex conju- 
gate transpose. We may point out here that when $\mathrm{S}$ is diagonal, i.e. when the impinging sources are uncorrelated, $\boldsymbol{R}$ will assume Toeplitz form.

Let the array size be increased by adding more sensors and group them into $\mathrm{K}$ overlapping subarrays, each of size $\mathrm{m}$. The first subarray is formed from the sensors $1, \mathbf{2}, \cdots, \boldsymbol{m}$ and the second subarray from $2,3, \cdots, \mathrm{m}+1$ and so on. Let $\mathbf{r}_{i}(\cdot)$ denote the vector of received signals from the th subarray. Then the ith subarray covariance matrix and the smoothed array covariance matrix $\bar{R}$ are given by

$$
\begin{gathered}
R_{i}=E\left[\mathbf{r}_{i}(t) \mathbf{r}_{i}^{+}(t)\right] \\
\bar{R}=\frac{1}{K} \sum_{i=1}^{K} R_{i}=A \bar{S} A^{+}+\sigma_{n}^{2} I
\end{gathered}
$$

where

$$
\bar{S}=\frac{1}{K} \sum_{n=1}^{K}\left\{\phi^{n-1} S \phi^{-(n-1)}\right\}
$$

and $\phi$ is a diagonal matrix

$$
\phi=\operatorname{diag}\left\{e^{-j w_{0} \tau_{1}}, \cdots, e^{-j w_{0} \tau_{d}}\right\} .
$$

Note that $\bar{S}$ is the smoothed source covariance matrix. With progressive smoothing, the nondiagonal terms of this matrix decrease and tend to zero asymptotically. (The overbar is used to denote quantities corresponding to the smoothed matrix.)

\section{A. Toeplitzization Algorithm of Takao et al. [1]}

In this weighted spatial smoothing scheme, the weights are chosen so as to make the smoothed matrix Toeplitz, and thus ensure perfect decorrelation of the sources. Deviation of the smoothed matrix $\overline{\boldsymbol{R}}$ from the Toeplitz form is defined as the sum of the squares of the absolute deviations of each element in $\overline{\boldsymbol{R}}$ from the along diagonal mean, parallel to the main diagonal. Since $\overline{\boldsymbol{R}}$ is Hermitian, only the main- and subdiagonals need be considered. The total deviation is thus given by

$$
\epsilon=\sum_{v=0}^{m-2} \sum_{x=1}^{m-v}\left|\bar{R}(x+v, x)-R_{\mathrm{av}}(v)\right|^{2}
$$

where

$$
R_{a v}(v)=\frac{1}{\mathrm{~m}-v} \sum_{z=1}^{m-v} \bar{R}(z+v, z)
$$

is the along diagonal mean.

The weight vector $\mathrm{f}$, is a K-length vector consisting of the weights for $\mathrm{K}$ subarrays, chosen so as to minimize this deviation subject to the condition that the sum of the weights is unity. The smoothed matrix can then be expressed as

$$
\bar{R}=\sum_{n=1}^{K} \mathbf{f}_{t}(n) R_{n} .
$$

We can write (10) as (see [1])

$$
\boldsymbol{\epsilon}=\mathbf{f}_{i}^{T} E \mathbf{f},
$$

where

$$
E=\operatorname{Re}\left\{\sum_{v=0}^{m-2} \sum_{x=1}^{m-v} \mathbf{e}_{x+v, x} \mathbf{e}_{x+v, x}^{+}\right\}
$$

with

$$
\mathbf{e}_{x+v, x}(n)=R_{n}(x+v, x)-\frac{1}{m-v} \sum_{z=1}^{m-v} R_{n}(z+v, z) .
$$

In the definition of $\mathrm{E}$, the real part is taken to ensure that $\mathrm{f}$, is a real vector.

To eliminate any possible numerical problems in inverting $\boldsymbol{E}$, Takao et al. [1] use diagonal loading and obtain the weight vector $f$,

$$
\mathrm{f},=\frac{(E+\eta I)^{-1} \mathbf{u}}{\mathbf{u}^{T}(E+\eta I)^{-1} \mathbf{u}}
$$

where $\mathbf{u}$ is a $\mathrm{K}$-length vector with each element equal to unity. If there are $\mathrm{d}$ fully correlated sources, we need to suppress $\left(d^{2}-d\right) / 2$ complex correlations requiring $\left(d^{2}-\mathbf{d}+1\right)$ constraints (including the unity constraint on the sum of the weights) to be satisfied. Since there are $\mathrm{K}$ weights, to fully Toeplitzize $\boldsymbol{R}$ we require

$$
K \geq d^{2}-d+1 \text {. }
$$

\section{B. Scheme of Paulraj et al. [2]}

In this method the sources are decorrelated by directly making the smoothed source covariance matrix $\bar{S}$ diagonal. The smoothed source covariance matrix, when we weight the subarray covariances with weights $f$, is given by

$$
\bar{S}=\sum_{n=1}^{K}\left\{\mathbf{f}_{p}(n) \phi^{n-1} S \phi^{-(n-1)}\right\}
$$

where $\phi$ is as defined in (9). The $(\boldsymbol{i}, \boldsymbol{t})$ element of $\overline{\boldsymbol{S}}$ can be written as

where

$$
\bar{S}(i, t)=S(i, t) \mathbf{f}_{p}^{+} \psi_{i t}
$$

$$
\psi_{i t}=\left[e^{j w_{0}\left(\tau_{t}-\tau_{i}\right)} e^{2 j w_{0}\left(\tau_{t}-\tau_{i}\right)}, \cdots, e^{j w_{0}(K-1)\left(\tau_{t}-\tau_{i}\right)}\right]^{T} .
$$

To force $\bar{S}$ to diagonal form, we require (noting that $\bar{S}$ is Hermitian)

$$
\bar{S}(i, t)=0, \quad t>i .
$$

From (19) and (21), it follows that the quantity $\mathbf{f}_{p}^{T} \psi_{i t}$ should be constrained to zero for all $\boldsymbol{t}>\boldsymbol{i}, \boldsymbol{i}, \boldsymbol{t}=1,2, \cdots, \mathbf{d}$. Since $\psi_{i t}$ is complex and f, is real, we need to force $\mathrm{f}_{\rho}^{T}\left(\boldsymbol{R} \boldsymbol{e}\left(\psi_{i t}\right)\right.$ and $\mathbf{f}_{p}^{T} \operatorname{Im}\left(\psi_{i t}\right)$ to zero separately. To make use of any extra degrees of freedom that may be available, the norm of the weighting coefficients is minimized. The weights $f$, are thus chosen as the solution of the minimization

$$
\begin{gathered}
\min \mathrm{f}_{\rho}^{T_{\mathrm{f}}} \\
\text { subject to } \mathrm{f}_{p}^{r_{\rho}} \Psi=[0,0, \cdots, 0]
\end{gathered}
$$

and

$$
\mathbf{f}_{p}^{T} \mathbf{u}=1
$$

where

$$
\begin{aligned}
\Psi=\left[\operatorname{Re}\left(\psi_{12}\right), \operatorname{Re}\left(\psi_{23}\right), \cdots, \operatorname{Re}\left(\psi_{d-1, d}\right),\right. \\
\left.\operatorname{Im}\left(\psi_{12}\right), \operatorname{Im}\left(\psi_{23}\right), \cdots, \operatorname{Im}\left(\psi_{d-1, d}\right)\right] .
\end{aligned}
$$

The solution to this linearly constrained minimization problem is given by

$$
\mathrm{f},=[\boldsymbol{\Psi} \mathbf{u}]\left\{[\boldsymbol{\Psi} \mathbf{u}]^{T}[\Psi \mathbf{u}]\right\}^{-1} \xi
$$

where $\xi$ is a $\left(d^{2}-\mathrm{d}+1\right)$ length vector with the last entry equal to unity and the rest as zeros

$$
\xi=\left[\begin{array}{lllll}
0 & 0 & \cdots & 0 & 1
\end{array}\right]^{T} .
$$




\section{Alternate Formulation}

We now suggest an alternate formulation for the above problem where we do not require a priori knowledge of the DOA's of the impinging sources and simultaneously use any excess available degrees of freedom to minimize the norm of the weighting coefficients. Thus, the alternate formulation is given as

$$
\begin{gathered}
\min \mathbf{f}_{a}^{T_{\mathbf{f}}}{ }_{a} \\
\text { subject to } \mathrm{f}_{\boldsymbol{a}}^{\boldsymbol{T}_{\boldsymbol{E}} \mathbf{E}},=0
\end{gathered}
$$

and

$$
\mathbf{f}_{a}^{T} \mathbf{u}=1 .
$$

$\mathbf{E}$ is a real symmetric matrix. Using spectral decomposition, we can write

$$
E=\sum_{i=1}^{d^{2}-d} \lambda_{i} \mathbf{y}_{i} \mathbf{y}_{i}^{T}
$$

Thus, $\mathbf{f}_{a}^{T} E \mathbf{f}_{a}=0$ is equivalent to

$$
\mathbf{f}_{a}^{T} \mathbf{y}_{i}=0, \quad i=1, \cdots,\left(d^{2}-d\right) .
$$

The formulation (26) can now be stated as a linearly constrained minimization problem

$$
\begin{gathered}
\min \mathbf{f}_{a}^{T_{\mathbf{f}}} \\
\text { subject to } \mathbf{f}_{a}^{\boldsymbol{T}} \boldsymbol{Y}=[0, O, \cdots, \mathbf{0}]
\end{gathered}
$$

and

$$
\mathbf{f}_{a}^{T} \mathbf{u}=1
$$

where $\boldsymbol{Y}$ is the matrix of the first $\left(d^{2}-\mathrm{d}\right.$ ) eigenvectors of $\mathbf{E}$ (those associated with nonzero eigenvalues);

$$
Y=\left[y_{1}, y_{2}, \cdots, y_{d^{2}-d}\right] \text {. }
$$

The solution to this problem is given by

$$
f,=[Y u]\left\{[Y u]^{T}[Y u]\right\}-{ }^{1} \xi .
$$

Note the similarity of this to the solution (24). Using block matrix inversion, (31) can be simplified to

$$
\mathbf{f}_{a}=\frac{\left(I-Y Y^{T}\right) \mathbf{u}}{\mathbf{u}^{T}\left(I-Y Y^{T}\right) \mathbf{u}}
$$

Now this looks similar to the solution (16).

We may point out here that when the impinging sources are uncorrelated, $\boldsymbol{E}$ reduces to the null matrix and the coefficients given by (16) correspond to the uniform weighting. We obtain a similar result from (32) if we note that $\boldsymbol{Y}$ is a null matrix when $\mathrm{E}$ is. We now explore the relationship between the weighting coefficients off the three methods (cf. (16), (24), and (32)).

IV. RelationshipbetweEn the ThreE Methods

From the definition of $\boldsymbol{E}$ (cf. (14)), we have

$$
\begin{aligned}
E= & \sum_{u=0}^{m-2} \sum_{x=1}^{m-u} \operatorname{Re}\left(\mathbf{e}_{x+v \cdot x} \mathbf{e}_{x+v, x}^{+}\right) \\
= & \sum_{u=0}^{m-2} \sum_{x=1}^{m-v}\left\{\operatorname{Re}\left(\mathbf{e}_{x+v, x}\right) \operatorname{Re}\left(\mathbf{e}_{x+v, x}^{+}\right)\right. \\
& \left.-\operatorname{Im}\left(\mathbf{e}_{x+v, x}\right) \operatorname{Im}\left(\mathbf{e}_{x+v, x}^{+}\right)\right\} .
\end{aligned}
$$

We can show (see Appendix) that

$$
\mathbf{e}_{x+v, x}=\sum_{p=1}^{d} \sum_{q=1}^{d} \frac{S(p, q) \delta_{p q}}{e^{j u w_{0} \tau_{p}}} \psi_{p q}
$$

where $\psi_{p q}$ is as defined in (20) and $\delta_{p q}$ is a scalar that depends on the spacing of the pth and qth sources, and is given by

$$
\delta_{p q}=\left\{\alpha_{p q}^{x-1}-\frac{\alpha_{p q}^{m-v}-1}{(m-v)\left(\alpha_{p q}-1\right)}\right\}
$$

with

$$
\alpha_{p q}=e^{j w_{0}\left(\tau_{q}-\tau_{p}\right)}
$$

Now (34) shows that $\mathrm{e}_{x+u, x}$ is a linear combination of $\psi_{p q}$, $\mathrm{p} \neq q$. Hence the space spanned by the corresponding vectors is also equivalent, i.e.,

$$
\begin{aligned}
\mathbf{\Omega}\left\{\mathbf{e}_{x+v, x} ; v=0 \cdots \mathbf{m}-2, x=1 \cdots \mathbf{m}-v\right\} \\
=\Omega\left\{\psi_{p q} ; \mathrm{P} \neq q, p, \mathbf{4}=1 \cdots d\right\}
\end{aligned}
$$

where $\Omega$ denotes the space spanned. This implies that

$$
\begin{aligned}
\boldsymbol{\Omega}\left\{\operatorname{Re}\left(\mathbf{e}_{x+v, x}\right), \operatorname{Im}\left(\mathbf{e}_{x+v, x}\right) ; v=0 \cdots \mathbf{m}-\mathbf{2}, \mathbf{x}=\mathbf{1} \cdots \mathbf{m}-\mathbf{v}\right\} \\
=\mathbf{R}\left\{\operatorname{Re}\left(\psi_{p q}\right), \operatorname{Im}\left(\psi_{p q}\right) ; \mathrm{P} \neq q, p, q=1 \cdots d\right\} \\
=\Omega\{\Psi\} .
\end{aligned}
$$

Combining (33) and (38), we have

$$
\begin{aligned}
\mathbf{\Omega}\{E\} & =\mathbf{\Omega}\left\{\operatorname{Re}\left(\mathbf{e}_{x+v, x}\right), \operatorname{Im}\left(\mathbf{e}_{x+v, x}\right)\right. \\
v & =0 \cdots m-2, x=1 \cdots m-v\} \\
& =\mathbf{R}\{\Psi\} .
\end{aligned}
$$

This shows that the rank of $\mathrm{E}$ is same as the rank of $\Psi$, which has $d^{2}-\mathrm{d}$ independent columns and hence a rank of $d^{2}-\boldsymbol{d}$. Also, since the space spanned by $\mathrm{E}$ is the same as the one spanned by its eigenvectors, we finally have

$$
\Omega\{E\}=\Omega\{Y\}=\Omega\{\Psi\}
$$

In view of (40), the orthogonality constraints in (22) and (29) are the same and since the problem formulation is identical, the solution is necessarily the same. Thus the weights obtained by the method of Paulraj et al. [2] are the same as those given by the alternate formulation (cf. (32)), i.e.,

$$
\mathbf{f}_{a}=\mathbf{f}, .
$$

This result is due to the fact that diagonalization of the smoothed source covariance matrix results in Toeplitzization of the array covariance matrix.

Now consider the solution of the method of Takao et al. [1], given by (16)

$$
\mathbf{f}_{t}=\frac{E_{1}^{-1} \mathbf{u}}{\mathbf{u}^{T} E_{1}^{-1} \mathbf{u}}
$$

with

$$
E_{1}=E+\eta I .
$$

Using eigendecomposition as in (27), we have

$$
E_{1}=\sum_{i=1}^{K}\left(\lambda_{i}+\eta\right) \mathbf{y}_{i} \mathbf{y}_{i}^{T}
$$


with

$$
\lambda_{i}=0, \quad i>d^{2}-d .
$$

Now, using simple manipulations we can show that

$$
\begin{aligned}
E_{1}^{-1} & =\sum_{i=1}^{K} \frac{1}{\left(\lambda_{i}+\eta\right)} \mathbf{y}_{i} \mathbf{y}_{i}^{T} \\
& =\sum_{i=1}^{d^{2}-d}\left\{\frac{1}{\left(\lambda_{i}+\eta\right)}-\frac{1}{\eta}\right\} \mathbf{y}_{i} \mathbf{y}_{i}^{T}+\frac{1}{\eta} I .
\end{aligned}
$$

Since $\eta$ is a small quantity, it is reasonable to assume that $\lambda_{i} \gg \eta$, $\boldsymbol{i}=1, \cdots, d^{2}-\boldsymbol{d}$. Neglecting the first term in the brackets and using (30), we get

$$
\begin{aligned}
E_{1}^{-1} & \approx \sum_{i=1}^{d^{2}=d}\left\{-\frac{1}{\eta}\right\} \mathbf{y}_{i} \mathbf{y}_{i}^{T}+\frac{1}{\eta} I \\
& =\frac{1}{\eta}\left(-Y Y^{T}+I\right) .
\end{aligned}
$$

Using this in (42) and comparing with (32), we obtain

$$
\mathrm{f}, \approx \frac{\left(\mathbf{I}-Y Y^{T}\right) \mathbf{u}}{\mathbf{u}^{T}\left(I-Y Y^{T}\right) \mathbf{u}}=\mathrm{f}_{a} .
$$

Combining (41) and (48), we have

$$
\mathrm{f}, \approx \mathbf{f}_{a}=\mathbf{f}_{p} \text {. }
$$

It is interesting to note that the weights given by the alternate formulation are essentially the same as those of the algorithm in [1], though the formulation in [1] does not explicitly minimize the norm of the weighting coefficients. To examine this, consider the weight vector given by (16). This is the solution of the minimization of $\mathbf{f}_{t}^{T} E_{1} \mathbf{f}_{t}$ subject to the unity constraint on the sum of the weighting coefficients. Thus, the minimization of

$$
\mathbf{f}_{t}^{T} E_{1} \mathbf{f}_{t}=\mathbf{f}_{t}^{T} E \mathbf{f},+\eta \mathbf{f}_{t}^{T} \mathbf{f}_{t}
$$

also involves the minimization of the norm of the weighting coefficients when excess degrees of freedom are available. This, however, requires that $\eta$ be small. If $\eta$ is large, the norm of the weight vector will be minimized at the expense of the lower decorrelation.

\section{CONCLUSION}

In this communication, we have briefly introduced two recently proposed weighted subarray covariance averaging schemes [1], [2] and suggested an alternative formulation for the same problem. We have brought out the relationship between all the three methods and shown that they yield the same solution vectors.

\section{APPENDIX}

In this Appendix, we give the steps involved in the derivation of (34). The $n$th subarray covariance matrix can be written as

$$
\boldsymbol{R},=A S_{n} A^{+}+\sigma_{n}^{2} I=\sum_{p=l}^{d} \sum_{q=1}^{d} \mathbf{a}\left(\theta_{p}\right) \mathbf{a}^{+}\left(\theta_{q}\right)+\sigma_{n}^{2} I
$$

where

$$
S_{n}=\phi^{n-1} S \phi^{-(n-1)}
$$

From the definition of (9), we have

$$
S_{n}(p, 4)=S(p, q) \alpha_{p q}^{n-1}
$$

where

$$
\alpha_{p q}=e^{j w_{0}\left(\tau_{q}-\tau_{p}\right)} .
$$

Combining (51), (53), and (3), we can write

$R_{n}(x+v, x)$

$$
=\sum_{p=1}^{d} \sum_{q=1}^{d} \frac{S(p, q) \alpha_{p q}^{x+n-2}}{e^{j v w_{0} \tau_{p}}}+\sigma_{n}^{2} I(x+v, x) .
$$

Using (54) in (15), we obtain

$$
\begin{aligned}
\mathbf{e}_{x+v, x}(n)=\sum_{p=1}^{d} \sum_{q=1}^{d} \frac{S(p, q) \alpha_{p q}^{n-1}}{e^{j v w_{0} \tau_{p}}} \\
\cdot\left\{\alpha_{p q}^{x-1}-\frac{1}{m-v} \sum_{z=1}^{m-v} \alpha_{p q}^{z-1}\right\} .
\end{aligned}
$$

Expression (34) then immediately follows from (55).

\section{REFERENCES}

[1] K. Takao and N. Kikuma, "An adaptive array utilising an adaptive spatial averaging technique for multipath environment," IEEE Trans. Antennas Propagat., vol. AP-35, pp. 1389-1396, Dec. 1987.

[2] A. Paulraj, V. U. Reddy, and T. Kailath, "Analysis of signal cancellation due to multipath in optimum beamformers for moving arrays," IEEE J. Ocean. Eng., vol. OE-12, pp. 163-172, Jan. 1987.

[3] R. O. Schmidt, "Multiple emitter location and signal parameter estimation," IEEE Trans. Antennas Propagat., vol. AP-34, pp. 276-280, Mar. 1986.

[4] J. Capon, "High-resolution frequency-wavenumber spectrum analysis," Proc. IEEE, vol. 57, pp. 1408-1418, Aug. 1969.

[5] T. J. Shan and T. Kailath, "Adaptive beamforming for coherent signals and interferences," IEEE Trans. Acoustics, Speech, Signal Processing, vol. ASSP-33, pp. 527-536, June 1985.

[6] T. J. Shan, M. Wax, and T. Kailath, "Spatial smoothing approach to location estimation of coherent sources," in Proc. 17th Asilomar Conf. Circuits, Systems and Computers, 1983, pp. 367-371.

[7] V. U. Reddy, A. Paulraj, and T. Kailath, "Performance analysis of the optimum beamformer in the presence of correlated sources and its behavior under spatial smoothing," IEEE Trans. Acoustics, Speech, Signal Processing, vol. ASSP-35, pp. 927-936, July 1987.

\section{On the Radial Component of the Electric Field for a Monopole Phased Array Antenna Focused in the Near Zone}

Alan J. Fenn

\begin{abstract}
The near-zone radial and principal spherical components of the electric field for a linear phased array antenna that is focused at one to two aperture diameters in the near zone are investigated. The phased array antenna consists of thin monopole elements, which are analyzed by using the method of moments. A theoretical formulation is described and computer simulation results are presented. The results show that the radial component is negligible in the focused near-field region, which allows the principal component to be accurately computed from knowledge of only the tangential electric field on the focal plane.
\end{abstract}

\section{INTRODUCTION}

The theoretical focused near-field adaptive nulling performance of phased array antennas has been studied recently, taking into account

Manuscript received February 25, 1991; revised December 23, 1991. This work was sponsored by the Department of the Air Force under Contract F19628-90-C-0002.

The author is with the Lincoln Laboratory, Massachusetts Institute of Technology, Lexington, MA 02173.

IEEE Log Number 9200426. 